\title{
Volitional control of anticipatory ocular smooth pursuit after viewing, but not pursuing, a moving target: evidence for a re-afferent velocity store
}

Received: 14 January 1997 / Accepted: 30 April 1997

\begin{abstract}
Although human subjects cannot normally initiate smooth eye movements in the absence of a moving target, previous experiments have established that such movements can be evoked if the subject is required to pursue a regularly repeated, transient target motion stimulus. We sought to determine whether active pursuit was necessary to evoke such an anticipatory response or whether it could be induced after merely viewing the target motion. Subjects were presented with a succession of ramp target motion stimuli of identical velocity and alternating direction in the horizontal axis. In initial experiments, the target was exposed for only $120 \mathrm{~ms}$ as it passed through centre, with a constant interval between presentations. Ramp velocity was varied from \pm 9 to $45^{\circ} \mathrm{s}$ in one set of trials; the interval between ramp presentations was varied from 640 to $1920 \mathrm{~ms}$ in another. Subjects were instructed either to pursue the moving target from the first presentation or to hold fixation on another, stationary target during the first one, two or three presentations of the moving display. Without fixation, the first smooth movement was initiated with a mean latency of $95 \mathrm{~ms}$ after target onset, but with repeated presentations anticipatory smooth movements started to build up before target onset. In contrast, when the subjects fixated the stationary target for three presentations of the moving target, the first movement they made was already anticipatory and had a peak velocity that was significantly greater than that of the first response without prior fixation. The conditions of experiment 1 were repeated in experiment 3 with a longer duration of target exposure (480 ms), to allow higher eye velocities to build up. Again, after three prior fixations, the anticipatory velocity measured at $100 \mathrm{~ms}$ after target onset (when visual feedback would be expected to start) was
\end{abstract}

not significantly different to that evoked after the subjects had made three active pursuit responses to the same target motion, reaching a mean of $20 \%$ s for a $50 \%$ s target movement. In a further experiment, we determined whether subjects could use stored information from prior active pursuit to generate anticipatory pursuit in darkness if there was a high expectancy that the target would reappear with identical velocity. Subjects made one predictive response immediately after target disappearance, but very little response thereafter until the time at which they expected the target to reappear, when they were again able to re-vitalise the anticipatory response before target appearance. The findings of these experiments provide evidence that information related to target velocity can be stored and used to generate future anticipatory responses even in the absence of eye movement. This suggests that information for storage is probably derived from a common pre-motor drive signal that is inhibited during fixation, rather than an efference copy of eye movement itself. Furthermore, a high level of expectancy of target appearance can facilitate the release of this stored information in darkness.

Key words Eye movement · Ocular pursuit . Anticipation · Humans

\section{Introduction}

Anticipatory smooth-pursuit eye movements of velocity greater than $3-4 \%$ s cannot normally be initiated by human subjects in the absence of a moving target (Heywood and Churcher 1971; Barnes et al. 1987; Kao and Morrow 1994). Yet it is known that in a number of circumstances such anticipatory movements must be made in order to overcome the time delays within the visual motion processing and thus allow the subject to maintain predictive control. Experiments have revealed that anticipatory eye movements can be generated if repeated presentation of the moving target is given (Becker and Fuchs 1985; Barnes et al. 1987; Boman and Hotson 
1988). Thus, although the subject responds to the first presentation of the moving target with a latency of some 100-125 ms, subsequent presentation results in the development of anticipatory responses that build up well before the target actually appears (Barnes and Asselman 1991; Boman and Hotson 1992; Kao and Morrow 1994). Even if the subject is well aware of the motion and timing of the target before it appears and starts to move, it does not seem possible to initiate such an anticipatory response without having made a prior response to a moving target. These findings led to the suggestion that the prior appearance and tracking of the target leads to the build up of an internal store of information, a form of short-term working memory, that can then be played out to form the anticipatory estimate under suitable control from a timing control mechanism or periodicity estimator (PE; Barnes et al. 1987). Evidence seemed to indicate that the information being stored for this purpose was related to the eye velocity generated, since the magnitude of the anticipatory component increased with the eye velocity generated (Barnes and Asselman 1991; Kao and Morrow 1994; Ohashi and Barnes 1996).

The concept of a memory system for pursuit had earlier been put forward by a number of authors (von Noorden and Mackensen 1962; Whittaker and Eaholtz 1982; Lisberger et al. 1987) in order to explain the observation that, when the target suddenly disappears, eye velocity may be sustained for a short period of time (Becker and Fuchs 1985), even though feedback of retinal error information has been removed. This concept was embodied in a number of models in which there was an internal positive feedback of an efference copy of eye velocity that had the useful attribute of stabilising the pursuit system (Robinson 1971; Yasui and Young 1975; Lisberger and Fuchs 1978). Although these simpler models could not realistically simulate the phase relationships of normal pursuit, the general concept of an efference copy system for pursuit has gained wide acceptance (see Leigh and Zee 1991). However, as a result of other experiments involving visual suppression of the vestibulo-ocular reflex (VOR), we were led to suggest that it is not actually a copy of eye velocity that is being stored, but a common drive signal that may have quite different characteristics to eye velocity itself (Barnes and Grealy 1992).

We have now examined the validity of the efference copy hypothesis by carrying out further experiments with the repeated transient presentation technique used previously to elicit anticipatory pursuit. But, instead of requiring the subject initially to pursue the moving target, we have instructed the subject to fixate on another, stationary target for a number of presentations of the moving target before making an attempt to follow the moving target. In this way, we sought to examine whether the subjects could use the afferent retinal velocity error information derived from the passage of the moving target to build up the internal velocity store and thus subsequently initiate an anticipatory response without having previously made any eye movement in response to the target motion.

\section{Materials and methods}

In experiments 1 and 2, subjects were seated $1.5 \mathrm{~m}$ in front of a tangent screen, with the head fixed by clamps to the side of the head. A computer-generated visual display was projected on to the screen by an overhead projector (Barco 400). The display consisted of two elements, a central fixation target in the form of a cross embedded in a circle subtending $50 \mathrm{~min}$ arc at the eye and a larger, moving rectangular section of grating. The grating subtended $10^{\circ}$ (height) $\times 5^{\circ}$ (width) at the eye and was composed of five vertically oriented stripes of spatial frequency 1 cycle/deg. A horizontal blank band of height $1^{\circ}$ separated the grating into two halves so that there was no local overlay of the fixation target on the grating display as it moved. The subject's horizontal eye movements were measured by an infra-red limbus tracking technique (Skalar Iris) with a resolution of 5-10 min arc. Recorded eye displacements were well within the range of linearity of the recording system (approx. $\pm 20^{\circ}$ ).

The grating was made to move across the screen at constant velocity in alternating directions in the horizontal axis. The underlying display motion was a triangular waveform, but the display was only presented to the subject during a brief period as it passed through the centre of the subject's field of view (Fig. 1A). In the control (0 fixation) condition the subject was instructed to actively pursue the display each time that it appeared, in the manner described in previous experiments (Barnes and Asselman 1991). In this condition the subject was asked to pursue the midpoint between the two halves of the grating display rather than a single target, but this made little if any difference to the response induced, as shown by previous experiments using perifoveal stimuli (Winterson and Steinman 1978; Barnes and Hill 1984). In the fixation conditions, the subjects were presented with the stationary fixation target during the first one, two or three presentations of the moving grating and were instructed to hold the eye fixed on that target. The fixation target then went off midway between the previous and succeeding presentations of the moving display (see Fig. 1B), and the subject was instructed to follow the target when it next appeared. The subjects were specifically not told anything about making anticipatory eye movements during this period and most were naive of the responses likely to be induced. Each of the fixation conditions was examined with a motion stimulus that was composed of five consecutive sectors. In each sector the underlying triangular waveform stimulus remained the same, with a number of cycles that varied in a randomized manner between 4 and 7 . At the transition between different sectors, the target was blanked out for one complete cycle (Fig. 1A).

In experiment 1 the frequency of the triangular wave remained constant at $0.39 \mathrm{~Hz}$ (inter-presentation interval $1280 \mathrm{~ms}$ ) but the velocity was changed between sectors in a randomized manner from \pm 9 to $\pm 45 \%$. The duration of target exposure was kept deliberately brief at $120 \mathrm{~ms}$. This was to enhance the effects of velocity build-up, which previous experiments had shown to be most evident for brief ( $<240 \mathrm{~ms}$ ) exposures of the moving target (Ohashi and Barnes 1996).

In experiment 2, the velocity of the target was kept constant throughout all five sectors, but the timing between presentations was changed by changing the frequency of the underlying triangular waveform from 0.25 to $0.8 \mathrm{~Hz}$ in a randomized order, whilst maintaining two presentations per cycle. The interval between presentations thus varied from 640 to $1920 \mathrm{~ms}$; the exposure duration was kept constant at $120 \mathrm{~ms}$. Experiments 1 and 2 were carried out on the same group of eight normal subjects.

In experiments 3 and 4 , we attempted to optimize the conditions for the subject to produce anticipatory movements of high velocity. We examined the effects of a longer exposure duration $(480 \mathrm{~ms})$, an increased inter-presentation interval $(2000 \mathrm{~ms})$ and higher velocity target motion (up to $50^{\circ} / \mathrm{s}$ ). This experiment was carried out with two small targets placed one immediately above the other. They were projected on to a semicircular screen via motor-controlled mirrors that enabled one target (coloured white) to be moved in the horizontal axis, whilst the other target (coloured green) was fixed and remained illuminated throughout the run. An 
Fig. 1A, B Examples of eye movements made in response to a regularly repeated, ramp target motion stimulus. Underlying target motion stimulus was a triangular waveform of frequency $0.39 \mathrm{~Hz}$. Target was illuminated twice per cycle for a duration of $120 \mathrm{~ms}$, as indicated by shutter signal. In $\mathbf{A}$, the subject pursued the target from its first appearance; whereas, in $\mathbf{B}$, the subject fixated a stationary target for the first three target presentations. Fast phase components have been removed from eye velocity signal $(P V E$ predictive velocity estimate made when expected target failed to appear)

\section{(A). 0 prior fixations}

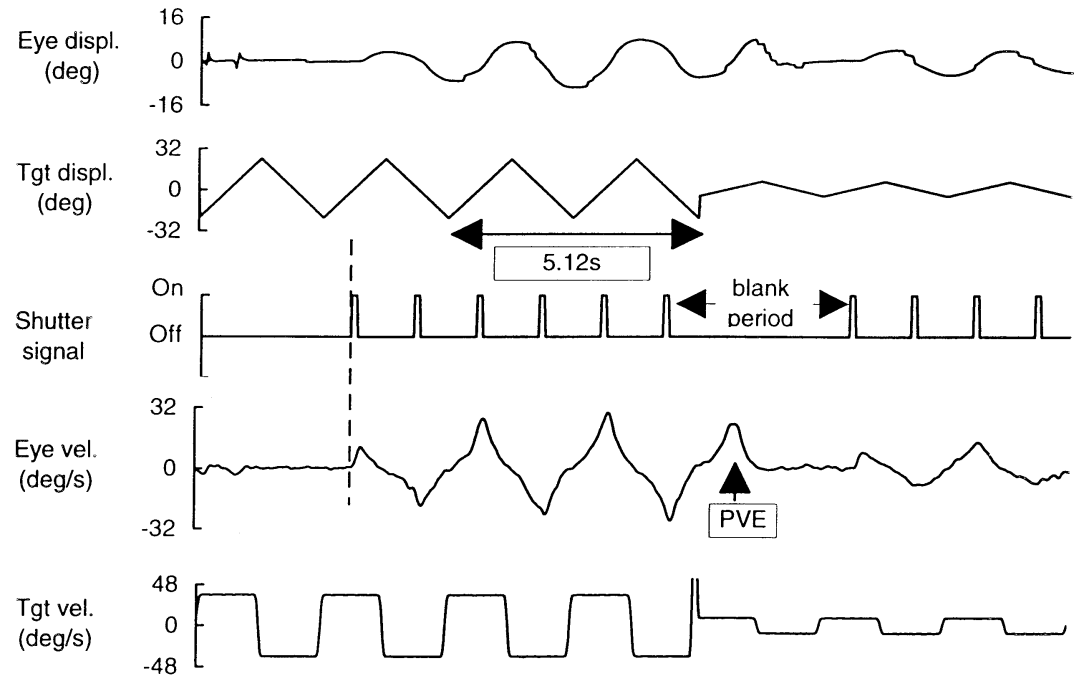

(B). 3 prior fixations.

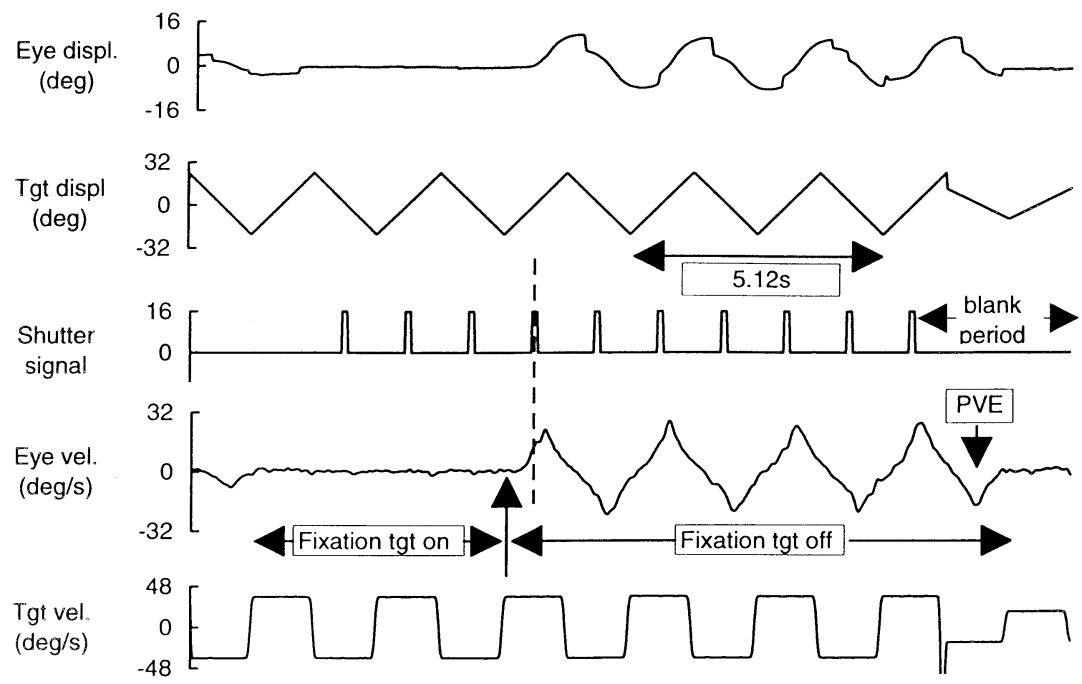

audio warning cue was given $760 \mathrm{~ms}$ prior to the start of each target presentation (i.e. at a time corresponding to peak displacement of the underlying waveform). An audio signal was also given during the presentation of the target to further facilitate the timing of eye movement responses. During blank periods, both audio signals remained on. Two stimulus conditions were examined.

In experiment 3 , the velocity was varied at random between the four sectors of the stimulus from 12.5 to $50 \%$ s. Subjects were instructed to fixate the stationary target for three presentations of the moving target by counting and then to use the fourth audio warning cue as a signal to initiate pursuit of the moving target during the next presentation. An important difference from experiments 1 and 2 was that the subjects were deliberately instructed to try to make the appropriate anticipatory eye movement after fixation by imagining the underlying motion of the target, which hitherto they had not tracked.

In experiment 4 , the target velocity remained the same $(50 \%)$ for all four successive sectors. In each sector, the target appeared intermittently for nine presentations (i.e. 4.5 stimulus cycles), but then failed to appear for the next three presentation periods (i.e. 1.5 stimulus cycles; Fig. 6). During this period of darkness, the subjects were instructed to continue making eye movements as if the target were still visible. After the period of three blank presentations, the target re-appeared with the same velocity and timing as in the previous sector. The audio cues were given continuously throughout all four sectors. The object was to determine how well subjects could sustain smooth eye movement in the blank periods and then use the velocity information obtained from the previous sector to generate an appropriate anticipatory response for the next sector. Experiments 3 and 4 were carried out by six subjects. All experiments were carried out with local ethical committee approval and the informed consent of the subjects.

Eye and target displacement signals were sampled at $100 \mathrm{~Hz}$ and digitally differentiated using a two-point difference algorithm. Saccadic eye movements were identified by an interactive, semiautomatic procedure, then removed and replaced by linear interpolation to obtain the smooth eye movement trajectories. As indicated in the examples shown in Fig. 1, the fast-phase movements were generally of small amplitude $\left(<5^{\circ}\right)$ and brief duration, making linear interpolation a simple and adequate method of waveform restoration. Various routines were then implemented on the derived velocity profiles to identify such features as the magnitude 
(A). 0 prior fixations.

Eye vel. (average)

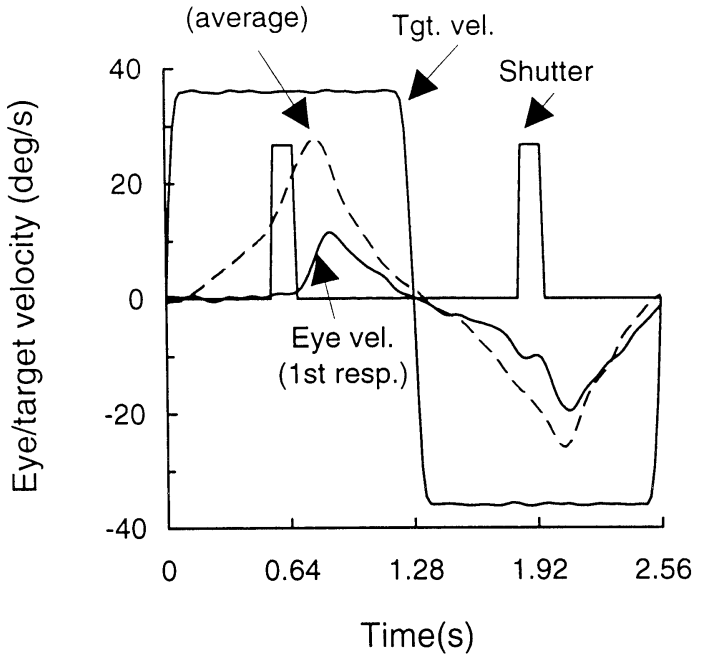

Fig. 2A, B Comparison of eye velocity trajectories shown in Fig. 1 for the first response made by the subject when there have been $\mathbf{A}$ no prior fixations or $\mathbf{B}$ three prior fixations. Mean eye velocity obtained from averaging of response to at least 2 cycles following initial response cycles

of peak velocity and the velocity $100 \mathrm{~ms}$ after target onset in response to each ramp stimulus. The time of onset of the eye velocity response to each target motion stimulus was calculated by identifying the point at which eye velocity reached a threshold equal to $10 \%$ of peak eye velocity, carrying out a linear regression on the next $100 \mathrm{~ms}$ of the velocity data and then extrapolating back to obtain the point at which the regression line crossed through zero. This was found to be a reliable way of identifying response onset for the predictive responses, for which eye velocity exhibited only a gradual increase with time. The response in each sector was analysed in two parts; a transient and a steady state phase. The transient phase comprised all initial presentations in which the subject was required to fixate the stationary target, plus the first two responses that were made to the moving target. The steady state response was represented by the remainder of the responses in that sector. Cycle-by-cycle means were obtained for the steadystate response by overlaying and averaging separately the left- and right-going responses. Statistical comparisons were carried out using repeated-measures analysis of variance (ANOVA).

\section{Results}

Effects of prior fixation on the anticipatory response

The most important finding from these experiments was that all subjects were able to make an anticipatory smooth eye movement to a previously observed moving target without having previously made an eye movement in pursuit of that moving target. Examples of this behaviour from experiment 1 are illustrated in Fig. 1B, in which the subject fixated on the stationary target for the first three presentations and then made an anticipatory response to the fourth target presentation. In contrast, Fig. 1A shows a typical response when there was no fixation period and the subject attempted to pursue the tar-
(B). 3 prior fixations.

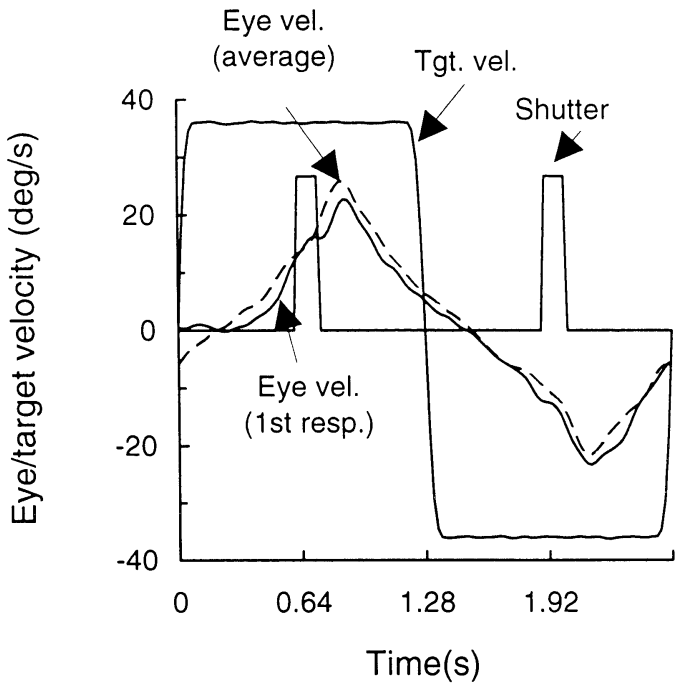

get from the first presentation. In both examples, after a variable number of presentations, the target failed to appear as expected, and the subject made a predictive velocity estimate (PVE) in the absence of the target, as noted previously (Barnes and Asselman 1991). After two such blank presentation periods, the subject made a new response to the different target motion stimulus, as shown in Fig. 1A. The differences between the fixation and non-fixation conditions are shown in more detail in Fig. 2, where the first response that the subject made has been compared with the mean (steady state) response for the remaining cycles of presentation. Without prior fixation (Fig. 2A), the smooth eye movement did not start until the target had been extinguished, that is, after a delay of approximately $120 \mathrm{~ms}$, whereas the mean response (broken trace) obtained from the remaining stimulus cycles exhibited an anticipatory smooth movement and a much higher peak velocity than that for the first response. In contrast, when three prior presentations of the target were allowed without pursuit (Fig. 2B), the subject was able to make an anticipatory response of high velocity after release of fixation that reached a peak velocity comparable with that of the mean response.

\section{Changes in peak eye velocity with prior fixation}

The objective of using a short target exposure (120 ms) in experiment 1 was that previous experiments (Barnes and Asselman 1991, Ohashi and Barnes 1996) had shown a build-up in peak velocity with repeated presentations that is not evident when longer exposure times are used. This build-up of peak velocity was observed in experiment 1 during the first four presentations in the no-fixation condition (Fig. 3B). The effect of presentation order was significant $\left(P<0.01 ; F_{3,140}=43.1\right)$ when tested by ANOVA, but it was notable that the mean peak velocity during the fourth presentation was greater than in the mean of subsequent steady state presentations (Fig. 3B). A similar, but more gradual, build- 
(A). 1st response.

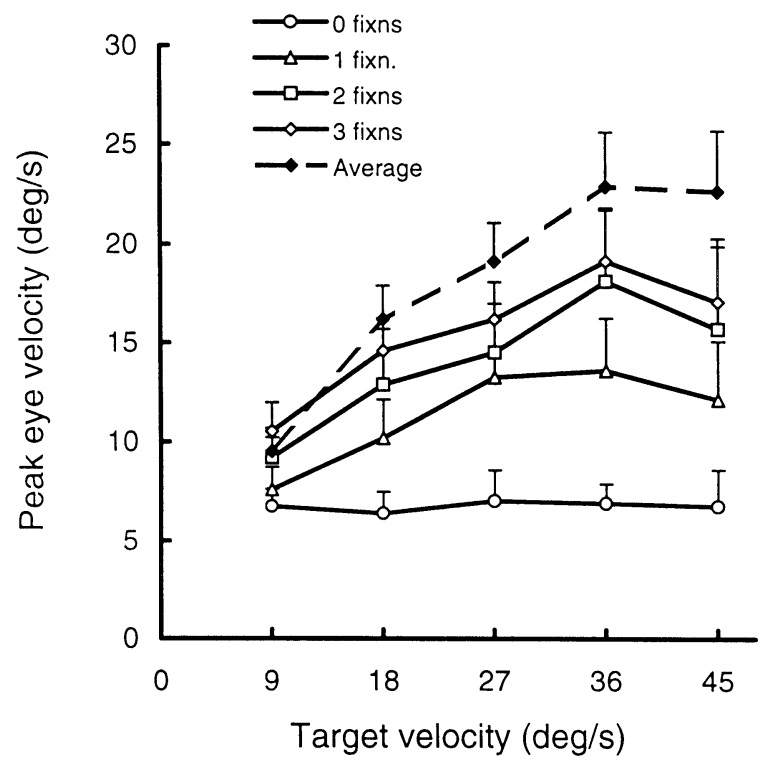

Fig. 3A, B Peak eye velocity as a function of target velocity during intermittent presentation of the moving target for an exposure period of $120 \mathrm{~ms}$. A The first response after $0,1,2$ or 3 presentations during which the subject fixated a stationary target. B The response made to the first four target presentations for the condition where there was no prior fixation ( 0 fixns in A). Broken line indicates mean steady state values of peak velocity. Mean of eight subjects + 1 SEM

up of peak velocity was also observed in the first response that the subject made as the number of fixation periods was increased (Fig. 3A). This effect also exhibited a significant trend with number of fixations $\left(P<0.001 ; F_{3,140}=12.6\right)$, although in this instance the mean velocity after three fixations did not quite reach the mean peak velocity in the subsequent steady state response. So, in other words, the peak velocity increased with the number of presentations whether or not the subject chose to pursue the target or merely to observe it whilst fixating, although the velocity achieved after fixation was somewhat less than that achieved during active pursuit. There was also a significant increase with increasing target velocity in both the fixation $\left(P<0.01 ; F_{4,140}=9.2\right)$ and non-fixation $\left(P<0.001 ; F_{4.140}=9.2\right)$ conditions, although post hoc one-way ANOVA showed there to be no significant change in velocity for the first response without fixation (common to both plots in Fig. 3).

Changes in the timing of anticipatory eye movements with prior fixation

In experiment 2 , when the subject made a normal, active pursuit response to the repeated presentation of the moving target without prior fixation, there was, in addition to the build-up of anticipatory velocity, a significant
(B). 0 prior fixations

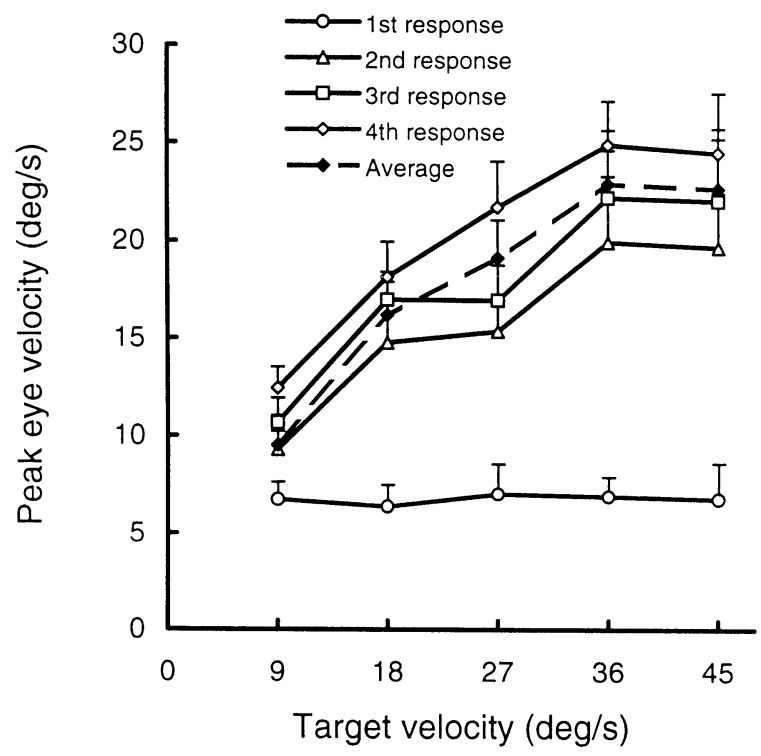

change $\left(P<0.001 ; F_{3,140}=46.2\right)$ in the timing of its initiation (Fig. 4A), as observed previously (Barnes and Asselman 1991). The first response that the subject made occurred with a mean latency (averaged across all interpresentation intervals) of $95 \mathrm{~ms}$ after target onset and was invariant with stimulus inter-presentation intervals. With repeated presentations, the response gradually occurred earlier with respect to the onset of target presentation. There was a significant difference $(P<0.01$; $F_{4,140}=4.2$ ) in latency with inter-presentation interval, the response being initiated earlier in relation to target onset when the interval was longer. However, at intervals of $1200 \mathrm{~ms}$ and greater, even after four responses, the subject had not attained the degree of anticipation that was observed in the averaged response derived from the fifth presentation onwards (broken line, Fig. 4A). The negative latency of this mean steady state response exhibited a highly significant decrease $\quad(P<0.001$; $F_{4,140}=471.9$ ) with increasing inter-presentation interval. For the longer intervals, there was a strong tendency to initiate the smooth movement at a time close to the equivalent of one-quarter of a cycle before the midpoint. In Fig. 4A, the time periods corresponding to one-quarter of a cycle before the midpoint have been shown for comparison. This effect is also evident in the averaged eye velocity profiles shown in Fig. 2, where it can be observed that the anticipatory response tends to start near the beginning of each half-cycle. It indicates that the subjects are initiating the smooth movement at a time when the target would have reached maximum displacement (or soon thereafter) and thus would be changing direction, although the subject could not actually see the moving target at this time.

When the subjects fixated on the stationary target during the first one, two or three presentations, the anticipatory smooth movement that they subsequently initiated occurred at approximately the same time as it would 
(A). 0 fixation periods

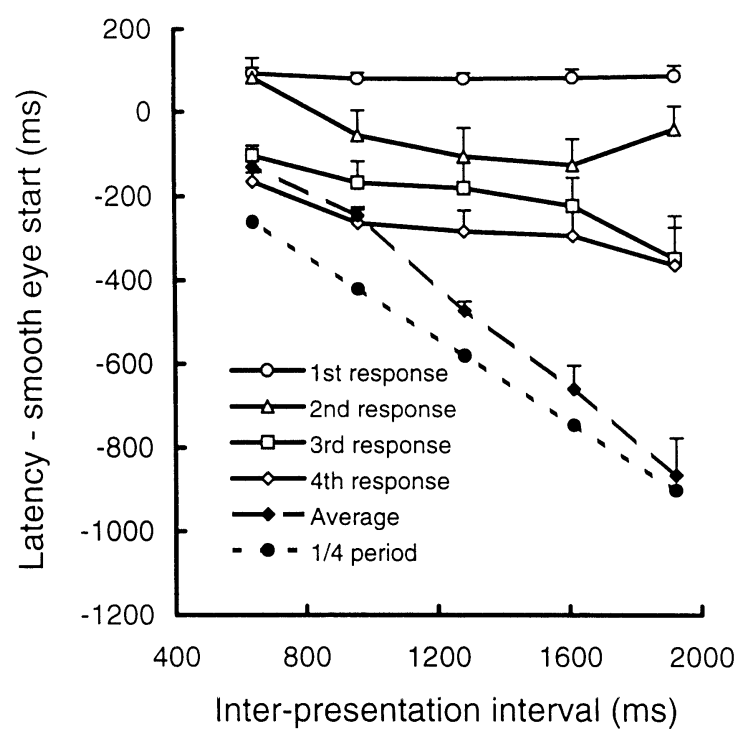

have done had the subject made an active pursuit response during those first presentations. This is illustrated in Fig. 4B for the two-fixation-period condition. In this condition there was still a significant increase in negative latency $\left(P<0.001 ; \mathrm{F}_{3,140}=61.5\right)$ with increasing number of active responses, but the timing of the first response that the subject made corresponded approximately in timing to the third response made when there was no fixation period. In fact, no significant difference was obtained when the third response with no fixation, the second response with one fixation and the first response with two fixations were compared by ANOVA. Similarly, there was no significant difference when the fourth response with no fixation was compared with the third with one fixation and the second with two fixations. Moreover, the third and fourth responses with two fixations were not significantly different in time of initiation from the mean steady state response, and, similarly, when three fixation periods were allowed, the second response that the subject made was initiated at a time that was not significantly different from the averaged response.

Changes in the magnitude of the anticipatory response after prior fixation

In experiment 3 , the results obtained with the higher velocity, longer duration ramps were similar to those obtained in experiment 1 in demonstrating that anticipatory smooth movements could be made after three prior fixations. The subjects were able to reliably use the timing information provided by the audio cue to initiate the response after counting three such cues, even though the fixation target remained on throughout. They were also able to use the much smaller moving target as effectively as the larger display used in experiment 1 . Figure $5 \mathrm{~A}$

\section{(B). 2 fixation periods}

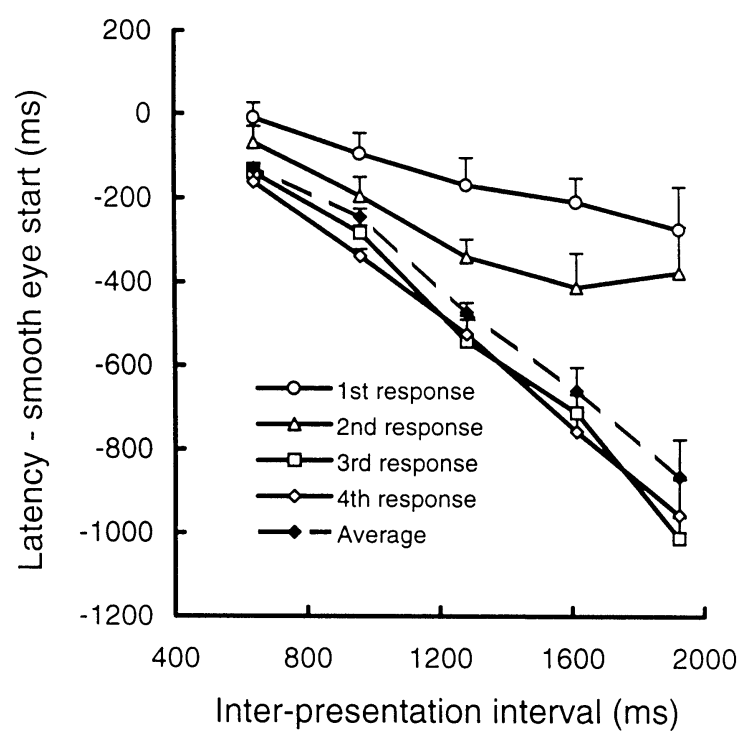

Fig. 4A, B The changes in timing of smooth eye movement onset in relation to target onset during the first four responses to an intermittently presented ramp target motion. In $\mathbf{A}$ there were no prior fixations of the stationary target; in $\mathbf{B}$ subjects had fixated the stationary target during two presentations of the moving target. Large dashed line indicates mean steady state values of timing. Mean of eight subjects +1 SEM. Small-dashed line shows $1 / 4$ period

shows the velocity trajectories of smooth eye movement for the first response made after three fixations and the mean response averaged over the remaining cycles of stimulation. These trajectories represent the averaged responses of all six subjects at one stimulus velocity $(37.5 \%)$, but the similarity between them was typical of that for the other stimulus velocities. There was considerable variability amongst subjects in the magnitude of response, but all were able to make anticipatory responses after three fixations in the manner indicated in Fig. 5A.

To compare magnitudes of the anticipatory response for different target velocities, eye velocity was calculated at target onset $\left(V_{0}\right)$ and $100 \mathrm{~ms}$ after target illumination $\left(V_{100}\right)$. As we have argued previously (Ohashi and Barnes 1996), any response made before $100 \mathrm{~ms}$ after target onset must represent an internally generated anticipatory response acting prior to the onset of visual feedback (Carl and Gellman 1987). Figure 5B shows the relationship between $V_{100}$ and the velocity of the target motion for the first response after three fixations. This has been compared with the value of $V_{100}$ obtained from the mean response for the subsequent six (steady state) target presentations. Analysis of variance indicated a significant increase $\left(P<0.01 ; F_{7,80}=2.98\right)$ in $V_{100}$ with target velocity, but no significant difference in $V_{100}$ between the first response after three fixations and the averaged steady state response. In other words, the anticipatory velocity build-up after three prior presentations 
(A).

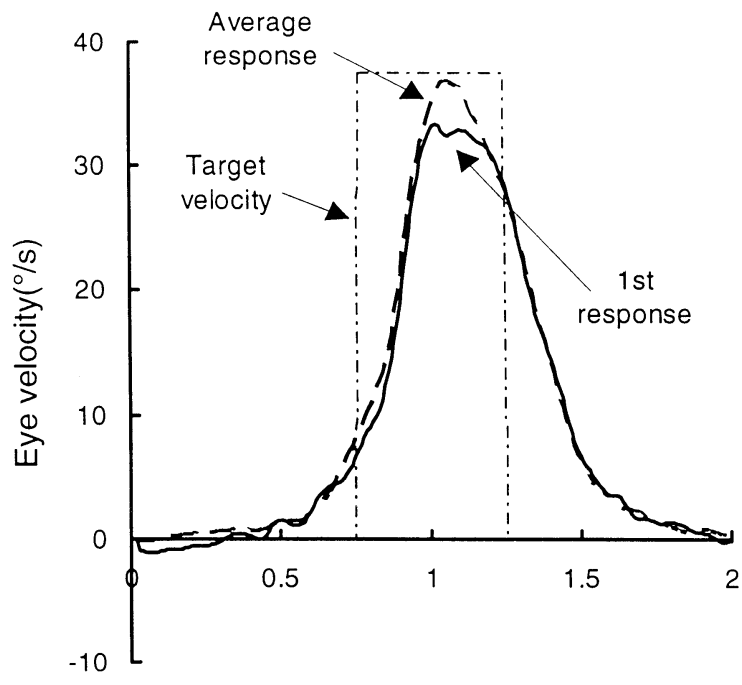

Time(s)

Fig. 5 A Comparison of the first smooth eye velocity trajectory made after three fixation periods with the mean (steady state) response to a target moving at $37.5 \%$ for an exposure period of $640 \mathrm{~ms}$. Mean of six subjects. Note the similarity in the anticipatory component. B. Comparison of the eye velocity $100 \mathrm{~ms}$ after target onset for the first response and the mean response to target velocities from 12.5 to $50 \%$ s. Mean of six subjects \pm 1 SEM

with fixation was graded in proportion to the expected target velocity in the same way as for the active pursuit responses. The velocity at onset $\left(V_{0}\right)$ was, on average, $22 \%$ less than $V_{100}$, but exhibited a similar significant trend $\left(P<0.01 ; F_{7,80}=3.5\right)$ with target velocity and no significant difference between the fixation and non-fixation conditions.
(B).

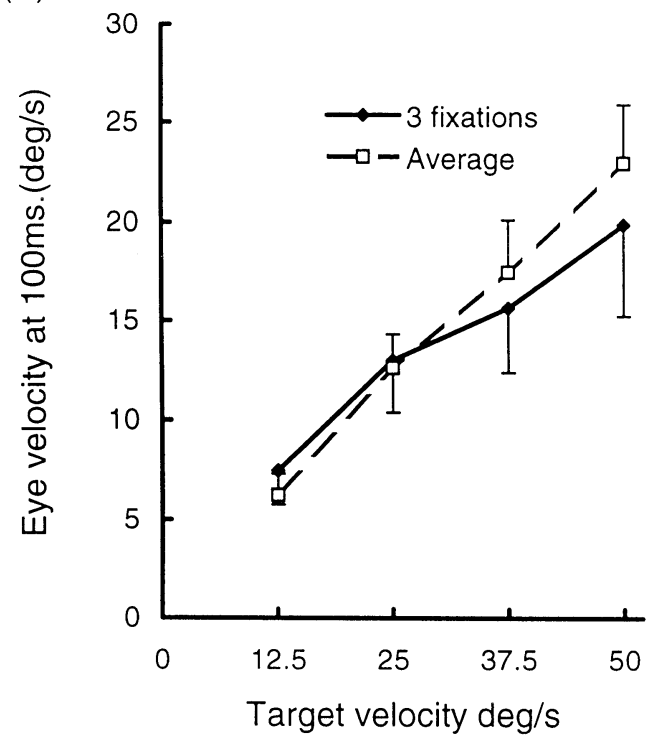

The effect of expectancy on the generation of anticipatory responses in the dark

In experiment 4 , the task for the subject was simply to fill in the gaps left by the three missing stimuli by making anticipatory movements at the times when they would be expected to occur (Fig. 6). With the audio cues, it was easy for the subjects to maintain the necessary rhythm, and they were fully aware that the target would be presented again after three blank periods. In these circumstances, all subjects made an anticipatory response during the first dark period (Fig. 6) at the end of each sector, as noted earlier (c.f. PVE in Fig. 1), with a mean value for $\mathrm{V}_{100}$ across all sectors of $16.9 \%$ s (Fig. 7B).
Fig. 6 Examples of eye movements from experiment 4 at the transition between sectors. In the "target off" period, the sound cues remain to indicate timing. The peak velocity of the eye progressively diminishes during successive half-cycles, but when the target is expected to reappear (at the beginning of the fifth half-cycle) the subject is able to initiate a full anticipatory response
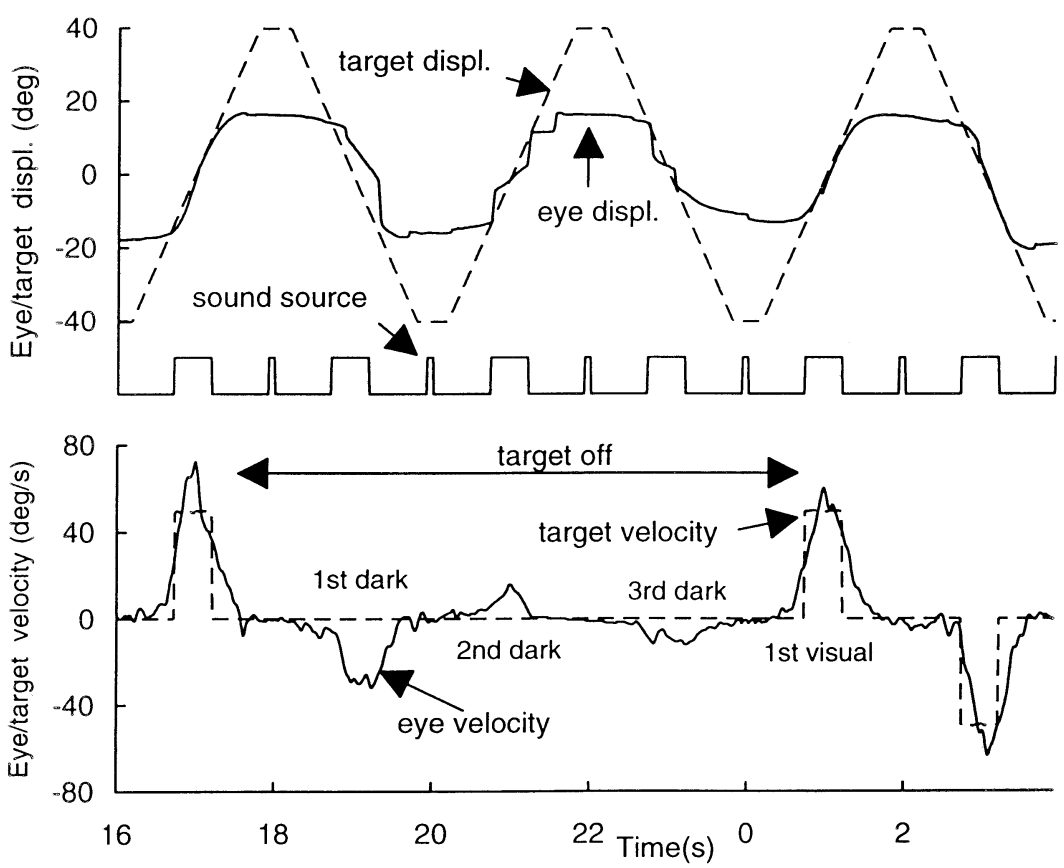
(A).

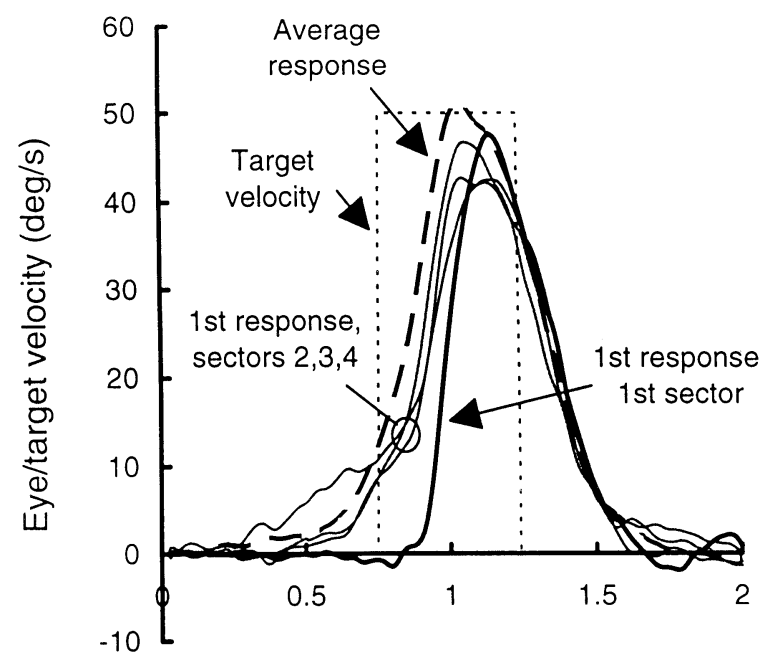

Time(s)
(B).

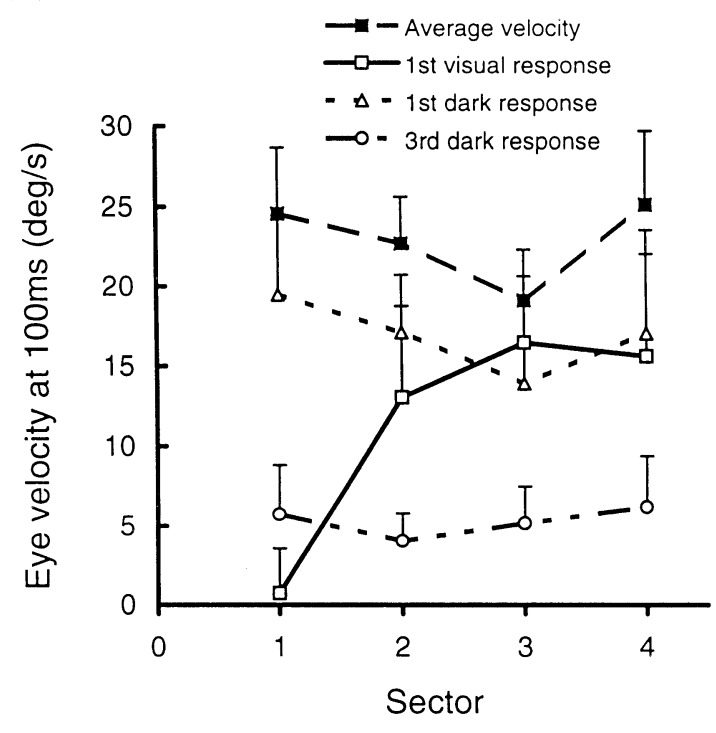

tors two to four with the first dark response of the immediately preceding sector (Fig. 7B) indicated no significant difference $\left(P=0.57 ; F_{1.48}=0.33\right)$ in the values of $V_{100}$. So, although the anticipatory smooth response appeared to have died out during the blank intervals, it was evident that the high level of expectancy that the target would reappear did allow the anticipatory response to be reproduced at a level that was comparable with the first preceding response in the dark.

\section{Discussion}

There can be little doubt now that humans do not normally possess the ability to initiate smooth eye movements of significant velocity $(>3-4 \%$ s) at will without a moving target, even in darkness. A large number of experiments have now demonstrated this (von Noorden and Makensen 1962; Heywood and Churcher 1971; Barnes et al. 1987; Kao and Morrow 1994), although it is acknowledged that some individuals do possess this ability (Heywood 1972). However, there can equally be no doubt that, given the right circumstances, all normal subjects are capable of generating smooth eye movements at will. One condition in which this ability is facilitated is when a stabilised image is presented on the retina in a perifoveal location (Kommerell and Taumer 1972; Grusser 1986; Barnes et al. 1995) and the subject directs attention to that location. The velocity of the smooth eye movement generated is dependent on the eccentricity of the centre of attention from the fovea, so that this appears to represent a form of positional feedback mediated by attention (Barnes et al. 1978; Pola and Wyatt 1980). Moreover, when the image is only partially stabilised on the retina so that it slides in the opposite direction to intended eye movement, subjects are still able to generate smooth movements, although they then become attenuated (Barnes et al. 1995). In this situation, it appears that the volitional effort is being opposed by the 
oppositely directed relative motion of the image on the retina. From this it has been suggested that the main reason that subjects cannot generate smooth movements at will across a stationary visual scene is because of the opposition provided by the optokinetic effect that would be produced if the eye were in motion.

Although these findings indicate that smooth eye movements can be generated at will, they do not offer an explanation for the fact that smooth movements cannot be initiated volitionally in the dark, a condition in which there should be no opposition to the internally generated movement. But it is possible to generate smooth eye movements of high velocity in the dark if the subject has previously pursued a moving target, even if there are intervening periods of darkness (Becker and Fuchs 1985; Barnes et al. 1987). As demonstrated by the results shown here (Fig. 1A), the first response to a transient target motion stimulus is normally initiated some $100 \mathrm{~ms}$ after target motion onset (Carl and Gellman 1987), but with repeated presentations subsequent responses become anticipatory (Fig. 4). Previously, we suggested that this effect arose from the storage and subsequent replay of the velocity information initially derived from the retinal error input during the first presentation. It has often been suggested that some form of velocity store must exist in order to allow eye velocity to be sustained during brief periods of target disappearance (von Noorden and Mackensen 1962). There is also evidence for storage of motion information that can be used for the perceptual comparison of one motion stimulus with another observed previously (Greenlee et al. 1995). Our own previous results (Barnes and Asselman 1991; Barnes and Grealy 1992) have shown that this store of velocity drive information can be held for considerable periods of time (up to $4 \mathrm{~s}$ at least) and then released, under the control of a PE, to form the anticipatory smooth eye movement that occurs prior to the next target appearance. As shown in Fig. 1, the appearance of the PVE at a time when the target was expected to appear, but failed to do so, appears to be a manifestation of the release of this stored information. Another demonstration of this storage ability can be observed when a previously tracked target motion trajectory is subsequently reproduced in the absence of that target motion stimulus through the use of a stabilised retinal image (Cushman et al. 1984; Van Den Berg and Collewijn 1987; Barnes et al. 1995).

The major new finding from the experiments described here is that the ability to store the velocity information required to generate anticipatory smooth movements does not depend on actually making an eye movement in pursuit of that moving target. Of particular importance is the demonstration that the magnitude and timing of the anticipatory response built up during fixation are appropriately scaled for the magnitude and timing of the target velocity stimuli. In experiment 3 , this anticipatory response (i.e. $V_{100}$ ) formed a relatively small part of the peak response, because from $100 \mathrm{~ms}$ after target onset the response was dominated by the effects of visual feedback. By contrast, in experiment 1 , the antici- patory response formed the major part of the peak velocity that could be attained, because the brief duration of target exposure allowed little to be added by visual feedback. Consequently there was still a clear difference in experiment 1 in the peak velocity attained after one, two or three periods of prior fixation (Fig. 3A). It is evident that the expectancy generated by viewing, but not pursuing, the moving target does not simply facilitate a generalised anticipatory response; rather, the unattended-to motion actually provides valid and useful quantitative information. But, it is also evident that expectancy does play a very large part in the ability to generate such anticipatory responses as demonstrated by the results of experiment 4 (Fig. 7). Kowler and colleagues (Kowler et al. 1984; Kowler 1989) have previously emphasised the importance of expectancy in relation to the determination of the direction of the anticipatory response, but the results presented here show a wider effect than this. Expectancy facilitates the generation of something that appears to have died out in the intervening period of darkness. Apart from this increased expectancy associated with the first appearance of the target in the next sector, there is no reason that the velocity response up until $100 \mathrm{~ms}$ after target onset should be any different to the corresponding responses initiated in darkness at the end of the previous sector.

A somewhat analogous situation to that of the fixation condition arises during visual suppression of the VOR (Barnes and Grealy 1992). When the subject is rotated on a turntable about the vertical axis so as to stimulate the lateral semicircular canals, smooth eye movements are generated that compensate for the head movement and thus tend to stabilise the eye in space. If the subject views a head-fixed target, these eye movements must be suppressed and a number of experiments have shown that stimulus features that limit the ability to suppress the VOR, such as the frequency of head oscillation and the predictability of stimulus motion, are very similar to those that limit ocular pursuit (Barnes et al. 1978; Lau et al. 1978; Lisberger et al. 1981; Barnes 1993). Of particular relevance is the observation that if the head-fixed target is exposed only briefly, but at regular intervals, in an analogous way to the target presentation in the pursuit experiments described here, a similar build-up of anticipatory suppression of the smooth (slow-phase) component of eye movement is observed (Barnes and Grealy 1992). But, in this instance, the effect of this anticipatory response is to reduce the velocity of the pre-existing VOR slow-phase, not to generate smooth eye movement as it does during pursuit.

Both pursuit and VOR suppression involve active use of the drive information. What is different and remarkable about the situation during fixation is that it appears that the anticipatory activity can be built up even when the subject is not using this information for active control but is attending to another task, that of fixating the stationary target. But it has been shown in previous experiments (Kowler and Steinman 1981; Barnes and Asselman 1991; Ohashi and Barnes 1996) that anticipatory 


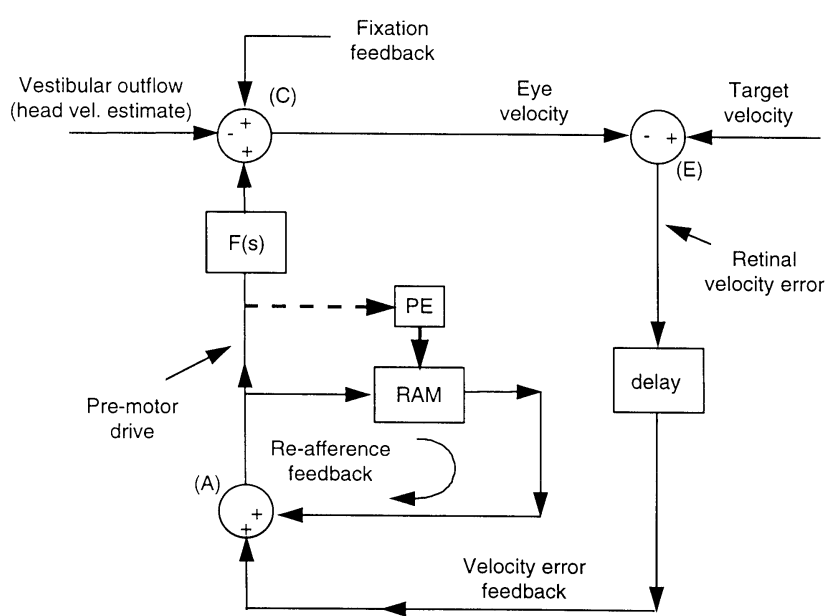

Fig. 8 A model of the interaction between the vestibulo-ocular reflex (VOR), fixation and pursuit mechanisms. Retinal error feedback derived from the moving target at junction $\mathrm{E}(E)$ forms the basic feedback mechanism for minimization of eye velocity error during pursuit and VOR suppression. The re-afferent feedback loop is responsible for construction of an internal representation of required oculomotor velocity drive by sampling the pre-motor drive signal and storing information in the re-afferent memory $(R A M)$. Timing of the output of RAM is controlled by the periodicity estimator $(P E)$ for summation with velocity error feedback at junction $\mathrm{A}(A)$ to form a new pre-motor drive signal. In ocular pursuit, pre-motor drive controls eye movement directly; during VOR suppression pre-motor drive inhibits the VOR at junction $\mathrm{C}(C)$; during fixation, pre-motor drive is inhibited at junction $\mathrm{C}$, but reafference feedback still allows pre-motor drive to be accumulated within $\operatorname{RAM}(F(s)$ - open-loop dynamics of visuomotor feedback)

smooth pursuit can be built up in this way even when the subject is not attending directly to the motion stimulus, but is stimulated passively by the movement of a background stimulus.

A simple way of representing all of this behaviour is through the type of model shown in Fig. 8, which is a simplified, adapted version of models described previously (Barnes and Asselman 1991; Barnes et al. 1995). The storage of velocity drive is achieved through the sampling and storage within the re-afferent memory (RAM) of a combination of current retinal error information summed (at junction A in Fig. 8) with prior output from the re-afferent positive feedback network. The output of this network thus initially represents retinal error information, but with repetition builds up to represent an internal estimate of the required pre-motor drive signal. The output of the store (RAM) is controlled by a PE that determines the correct time at which to release the contents of the store to form an anticipatory response. This model is really an elaboration of the original concept put forward by Yasui and Young (1975) and subsequently elaborated by a number of other authors (Lisberger and Fuchs 1978; Robinson 1982). But, previously, it has been suggested that the input for the velocity store comes from an efference copy of the eye velocity drive signal. It is evident from the experiments described here that it is certainly not necessary for the input to this store to be a copy of the eye movement itself, but is more likely to be corollary discharge from the pre-motor drive signal. The common feature of pursuit and VOR suppression is that the anticipatory activity is built up in the re-afferent network and can then be used either to drive the eye directly during ocular pursuit or to inhibit (at junction $\mathrm{C}$ in Fig. 8) the output of the vestibular system during VOR suppression. Similarly, during fixation of a stationary target, retinal error information derived from the fixation target would be used to suppress the oculomotor drive at junction C (Fig. 8). Input to the re-afferent store (RAM) obtained from velocity error feedback would still be maintained and allow charging of the store despite the suppression of eye movement resulting from fixation. The stored information could then be released once fixation was terminated to form a fully developed anticipatory response of the form observed experimentally (Fig. 5A).

An important implication of the findings presented here is that it may be possible to store anticipatory activity from sources of motion stimuli other than those currently being attended to, thus ensuring that time delays in pursuit are minimized when switching attention from one moving object to another. There is no doubt that the anticipatory smooth eye movement forms a very significant part of the normal oculomotor response, allowing peak eye velocity to be attained some $50 \mathrm{~ms}$ earlier than for a non-predictable stimulus (Kowler and Steinman 1979 ; Ohashi and Barnes 1996). The ability to store and retrieve, on demand, such anticipatory activity would thus carry a considerable advantage for survival.

\section{References}

Barnes GR (1993) Visual-vestibular interaction in the control of head and eye movement: the role of visual feedback and predictive mechanisms. Prog Neurobiol 41:435-472

Barnes GR, Asselman PT (1991) The mechanism of prediction in human smooth pursuit eye movements. J Physiol (Lond) 439: 439-461

Barnes GR, Grealy MA (1992) Predictive mechanisms of headeye coordination and vestibulo-ocular reflex suppression in humans. J Vestib Res 2:193-212

Barnes GR, Hill T (1984) The influence of display characteristics on active pursuit and passively induced eye movements. Exp Brain Res 56:438-447

Barnes GR, Benson AJ, Prior ARJ (1978) Visual-vestibular interaction in the control of eye movement. Aviat Space Environ Med 49:557-564

Barnes GR, Donnelly SF, Eason RD (1987) Predictive velocity estimation in the pursuit reflex response to pseudo-random and step displacement stimuli in man. J Physiol (Lond) 389: 111-136

Barnes GR, Goodbody SJ, Collins S (1995) Volitional control of anticipatory ocular pursuit responses under stabilized image conditions in humans. Exp Brain Res 106:301-317

Becker W, Fuchs AF (1985) Prediction in the oculomotor system: smooth pursuit during transient disappearance of a visual target. Exp Brain Res 57:562-575

Boman DK, Hotson JR (1988) Stimulus conditions that enhance anticipatory slow eye movements. Vision Res 28:1157-1165

Boman D, Hotson J (1992) Predictive smooth pursuit eye movements near abrupt changes in motion direction. Vision Res 32: 675-689

Carl JR, Gellman RS (1987) Human smooth pursuit: stimulus-dependent responses. J Neurophysiol 57:1446-1463 
Cushman WB, Tangney JF, Steinman RM, Ferguson JL (1984) Characteristics of smooth eye movements with stabilized targets. Vision Res 24:1003-1009

Greenlee MW, Lang HJ, Mergner T, Seeger W (1995) Visual short term memory of stimulus velocity in patients with unilateral posterior brain damage. J Neurosci 15:2287-2300

Grusser O (1986) The effect of gaze motor signals and spatially directed attention on eye movements and visual perception. In: Freund H, Buttner U, Cohen B, Noth J (eds) The oculomotor and skeletal-motor systems: differences and similarities. Elsevier, Amsterdam, pp 391-404

Heywood S (1972) Voluntary control of smooth eye movements and their velocity. Nature 238:408-410

Heywood S, Churcher J (1971) Eye movements and the afterimage. 1. Tracking the afterimage. Vision Res 11:1163-1168

Kao GW, Morrow MJ, Le HM (1994) The relationship of anticipatory smooth eye movements to smooth pursuit initiation Vision Res 34:3027-3036

Kommerell G, Taumer R (1972) Investigations of the eye tracking system through stabilized retinal images. In: Bizzi E (ed) Bibliotheca Ophthalmologica. Karger, Basel, pp 288-297

Kowler E (1989) Cognitive expectations, not habits, control anticipatory smooth oculomotor pursuit. Vision Res 29:1049-1057

Kowler E, Steinman RM (1979) The effect of expectations on slow oculomotor control. II. Single target displacements. Vision Res 19:633-646

Kowler E, Steinman R (1981) The effect of expectations on slow oculomotor control. 3. Guessing unpredictable target displacements. Vision Res 21:191-203

Kowler E, Martins A, Pavel M (1984) The effect of expectations on slow oculomotor control. 4. Anticipatory smooth eye movements depend on prior target motions. Vision Res 24:197-210

Lau CGY, Honrubia V, Jenkins HA, Baloh RW, Yee RD (1978) Linear model for visual-vestibular interaction. Aviat Space Environ Med 49:880-885

Leigh RJ, Zee DS (1991) The neurology of eye movements. Davis, Philadelphia
Lisberger SG, Fuchs AF (1978) Role of primate flocculus during rapid behavioural modification of vestibuloocular reflex. I. Purkinje cell activity during visually guided horizontal smoothpursuit eye movements and passive head rotation. J Neurophysiol 41:733-763

Lisberger SG, Evinger C, Johanson GW, Fuchs AF (1981) Relationship between eye acceleration and retinal image velocity during foveal smooth pursuit in man and monkey. J Neurophysiol 46:229-249

Lisberger SG, Morris EJ, Tychsen L (1987) Visual motion processing and sensory-motor integration for smooth pursuit eye movements. Annu Rev Neurosci 10:97-129

Noorden GK von, Mackensen G (1962) Pursuit movements of normal and amblyopic eyes. Am J Ophthalmol 53:325-336

Ohashi N, Barnes GR (1996) A comparison of predictive and nonpredictive ocular pursuit under active and passive stimulation conditions in humans. J Vestib Res 6:261-276

Pola J, Wyatt HJ (1980) Target position and velocity: the stimuli for smooth pursuit eye movements. Vision Res 20:523-534

Robinson DA (1971) Models of oculomotor neural organisations. In: Bach-y-Rita P (ed) The control of eye movements. Academic Press, New York

Robinson DA (1982) A model of cancellation of the vestibuloocular reflex. In: Lennerstrand G, Zee DS Keller EL (eds) Functional basis of ocular motility disorders. Pergamon Press, Oxford, pp 5-13

Van den Berg A, Collweijn H (1987) Voluntary smooth eye movements with foveally stabilized targets. Exp Brain Res 68: 195-204

Whittaker SG, Eaholtz G (1982) Learning patterns of eye motion for foveal pursuit. Invest Ophthalmol Vis Sci 23:393-397

Winterson BJ, Steinman RM (1978) The effect of luminance on human smooth pursuit of perifoveal and foveal targets. Vision Res 18:1165-1172

Yasui S, Young LR (1975) Perceived visual motion as effective stimulus to pursuit eye movement system. Science 190:906-908 